\title{
Problemáticas de la docencia universitaria ecuatoriana en tiempos de COVID-19
}

\section{Problems of Ecuadorian university teaching in times of COVID-19}

Rosa Marianela Salamea Nieto

Universidad Técnica de Machala, Ecuador

rsalamea@utmachala.edu.ec

iD https://orcid.org/0000-0001-5315-3439

Sandra Elizabeth Campoverde Romero

Universidad César Vallejo, Perú

sandracampoverde23@hotmail.com

(i) https://orcid.org/0000-0002-6099-1964

Luis Fernando Cedillo Chalaco

Universidad Técnica de Machala, Ecuador

lfcc_lye@hotmail.com

(i) https://orcid.org/0000-0002-3142-4485

John Alexander Campuzano Vásquez

Universidad Técnica de Machala, Ecuador

jcampuzano@utmachala.edu.ec

iD https://orcid.org/0000-0002-3901-3197

Recepción: 07/01/2021 | Aceptación: 03/02/2021 | Publicación: 10/05/2021

Cómo citar (APA, séptima edición):

Salamea Nieto, R. M., Campoverde Romero, S. E., Cedillo Chalaco, L.F., y Campuzano

Vásquez, J. A. (2021). Problemáticas de la docencia universitaria ecuatoriana en tiempos de

COVID-19. Innova Research Journal, 6(2), 105-116.

https://doi.org/10.33890/innova.v6.n2.2021.1674

\section{Resumen}

El coronavirus (COVID-19), ha sumido a los países del mundo en un apuro, no solo sanitario y económico, sino también educativo, que ha llevado a muchas naciones a la suspensión de las actividades docentes tradicionales. Ecuador es parte de esta realidad, su sistema universitario está inmerso en una urgente transición de las clases presenciales a formatos online. La Universidad Técnica de Machala es parte de este crítico momento, el cual ha generado varias problemáticas 
para los docentes, que de forma abrupta y en un período inusual los ha llevado a migrar sus clases a un formato educativo distinto al tradicional, constituyéndose un salto apresurado a la universidad del nuevo siglo, en donde la tecnología pasó de ser una herramienta esporádica a convertirse en una parte fundamental en la cátedra, marcando un nuevo nivel de fatiga y estrés en los educadores. Esta investigación usó una encuesta online que recogió las respuestas de docentes de la Universidad Técnica de Machala, para identificar el nivel de fatiga y estrés por el cual están atravesando por la virtualización de sus clases, debido a la pandemia del covid-19, obteniendo datos respecto a las problemáticas presentadas (logísticas, tecnológicas, pedagógicas y socioafectivas), y la edad (intervalos) de cada uno de ellos, los que son analizados usando un modelo ANOVA de doble vía. Los principales resultados muestran que el nivel de fatiga y estrés en los docentes universitarios se ha visto incrementado, y marca diferencias estadísticamente significativas respecto a las edades, y no significativas en cada problemática manifestada.

Palabras claves: ANOVA; COVID-19; educación universitaria; fatiga y estrés.

\begin{abstract}
The coronavirus (covid-19), has plunged the countries of the world into a hurry, not only health and economic, but also educational, which has led many nations to the suspension of traditional teaching activities. Ecuador is part of this reality, its university system is immersed in an urgent transition from face-to-face classes to online formats. The Technical University of Machala is part of this critical moment, which has generated several problems for teachers, which abruptly and in an unusual period has led them to migrate their classes to an educational format other than the traditional one, constituting a hasty leap to the university of the new century, where technology went from being a sporadic tool to becoming a fundamental part of the chair, marking a new level of fatigue and stress in educators. This research used an online survey that collected the responses of teachers from the Technical University of Machala, to identify the level of fatigue and stress through which they are going through the virtualization of their classes, due to the covid-19 pandemic, obtaining data Regarding the problems presented (logistical, technological, pedagogical and socio-affective), and the age (intervals) of each of them, which are analyzed using a two-way ANOVA model. The main results show that the level of fatigue and stress in university teachers has increased, and marks statistically significant differences with respect to age, and not significant in each problem manifested.
\end{abstract}

Keywords: ANOVA; COVID-19; university education; fatigue and stress.

\title{
Introducción
}

La pandemia del coronavirus (covid-19) se está propagando de forma exponencial en todos los países del mundo. Desde su aparición en Wuhan en diciembre de 2019, hasta la actualidad ${ }^{1}$, los casos confirmados reportados suman cerca de 23,4 millones; siendo Estados Unidos, el país que se ha convertido en el foco de la pandemia, seguido por Brasil y la India. A nivel de Latinoamérica, Ecuador, desde el 29 de febrero de 2020 que el Ministerio de Salud Pública (2020) anuncia el primer caso confirmado de coronavirus dentro del país, ocupa el octavo lugar en el número de contagiados, y el vigésimo octavo a nivel del mundo.

\footnotetext{
${ }^{1}$ Con corte a 24 de agosto de 2020, 23:00 horas- Ecuador. Datos proporcionados por (Google Noticias, 2020).
} 
Además, el COVID-19 resultó en una agravante para la crisis mundial, debido a las paralizaciones de actividades <<como medida drástica para desacelerar los niveles de contagios y muertes >>, sumiendo a los países del mundo en un apuro, no solo sanitario y económico (Banco Mundial, 2020), sino también educativo, que ha llevado a nivel global a la suspensión de las actividades docentes tradicionales, para migrar de manera abrupta al modelo de la educación online (Mora, Julio, Calderón, y Huilcapi, 2020), constituyéndose en una experiencia altamente complicada para estructurar una enseñanza educativa satisfactoria (Trujillo, Fernández, Segura, y Jiménez, 2020).

En concordancia con el inciso anterior, este crecimiento de contagios obligó a todos los países del globo terráqueo a tomar medidas de aislamiento y distanciamiento social, con el propósito de frenar su evolución y el acelerado acrecentamiento de muertes registradas; la medida que más destacó fue la suspensión de actividades educativas presenciales (García, Corell, Abella, y Grande, 2020), obligando a pasar a la educación virtual; un proceso, que si bien es cierto, se ha ido dando de manera progresiva en las instituciones de educación superior, representando para algunos docentes serias dificultades en el proceso de enseñanza (Martínez y Garcés, 2020).

Entre las dificultades evidenciadas por parte de la suspensión de actividades educativas presenciales, según una investigación llevada a cabo por (Pedró, 2020) en el contexto de América Latina y El Caribe, destaca principalmente impactos en lo pedagógico, financiero, laboral, movilidad internacional, sobre la demanda y oferta de educación, y socioemocional. En lo que refiere a este último aspecto, los principales rasgos que presentan los docentes por el traslado a la educación virtual de sus contenidos son: la ansiedad, nerviosismo, la fatiga y el estrés (Gómez y Rodríguez, 2020).

Según Fernández, Hernández, Nolasco, de la Rosa, y Herrera (2020) más de 120 países se han visto en la necesidad de cerrar de manera total o parcial sus sistemas educativos (públicos y privados) en el nivel de educación superior (como en el resto de niveles), resultando en una decisión nada fácil, debido a que esto implica múltiples costes para el sector de la educación, tales como: costes educativos, que se generan por llevar la educación tradicional a una educación virtual; costes sociales, desde el sentido de la reorganización para adaptarse a la "nueva" modalidad de estudio, y de que no todos los implicados tengan las posibilidades o condiciones para desenvolverse adecuadamente o acceder a ella; y costes laborales, para pagar a docentes sin que estos cumplan una productividad laboral regular.

Alania, Llancari, de la Cruz, y Ortega (2020) manifiestan que no resulta nada fácil para los docentes transitar de la educación tradicional dictada en las aulas a la educación virtual, por razones que dicha transición les significa buscar e implementar nuevas estrategias didácticas, tanto a nivel sincrónico como asincrónico, sumándose a su vez, en muchos de los casos la deficiencia de internet, que en conjunto generan dentro del proceso pedagógico situaciones de frustración, como el estrés y la fatiga, por los esfuerzos cognitivos y conductuales.

A raíz de lo descrito, y al ser Ecuador parte de esta realidad, su sistema educativo universitario se ha visto inmerso también en una urgente transición de las clases presenciales a formatos virtuales (Villafuerte, Johanna, Cevallos, y Bermello, 2020). Tal es el caso de La Universidad Técnica de Machala (como las otras universidades ecuatorianas) la cual es parte de 
este crítico momento educativo; el cual ha significado que a su planta docente le toque de forma abrupta y dentro de un período inusual migrar a un formato educativo distinto al tradicional, que aunque constituye un salto apresurado a la universidad del nuevo siglo, en donde la tecnología pasó de ser una herramienta esporádica a convertirse en una parte fundamental en el cumplimiento de la cátedra, y sumándose las problemáticas inmiscuidas en los procesos de diseños de syllabus, planes de clases, recursos y estrategias, han marcado un nuevo nivel de fatiga y estrés, con afectaciones directas a la estabilidad del educador.

Por tanto, se da la importancia de efectuar esta investigación, la cual tiene como objetivo, identificar el nivel de fatiga y estrés por el cual están atravesando los docentes universitarios de las Universidad Técnica de Machala por la virtualización de sus clases, debido a la pandemia del COVID-19, usando un modelo ANOVA de doble vías, estructurados con datos respecto a las problemáticas presentadas (logísticas, tecnológicas, pedagógicas y socio-afectivas), y la edad (intervalos) de cada uno de ellos.

\section{Metodología}

\section{Muestra}

Se empleó una técnica de muestreo no probabilístico por conveniencia (Otzen y Manterola, 2017), a los docentes investigadores de la UTMACH, a los que se les aplicó una encuesta online mediante Google forms, con la finalidad de obtener información respecto al nivel de fatiga y estrés por el cual están atravesando debido a la migración de sus contenidos de educación presencial a la educación virtual, por motivos de la pandemia del COVID-19; además, se plantearon preguntas que permitieron obtener datos respecto a las problemáticas presentadas (logísticas, tecnológicas, pedagógicas y socio-afectivas), y la edad de cada uno de ellos. Dicha encuesta se envió al total de docentes que están adscritos al departamento de investigación de la universidad, de los cuales únicamente 40 estuvieron disponibles para responder.

\section{Variables}

Se tomaron 3 variables para estructurar el modelo de ANOVA de dos vías:

> Variable dependiente nivel de fatiga y estrés: medida con un valor de 1 a 10 , en donde 1 significa muy bajo nivel de fatiga y estrés; y 10 muy alto nivel de fatiga y estrés.

> Factor (vía) problemáticas: logísticas, tecnológicas, pedagógicas y socio afectivos.

$>$ Factor (vía) edad: la edad de los participantes en intervalos. 27-31, 32-36, 37-41, 42-46, y $47+$.

\section{Análisis estadístico}

Para el curso investigativo se planteó un estudio cuantitativo descriptivo (Rubio, 2016), de corte transversal (Rodríguez y Mendivelso, 2018). En paralelo, se construyó un modelo de análisis de varianza (ANOVA) de dos vías, el cual busca determinar la existencia de diferencias entre las cuatro problemáticas planteadas (logísticas, tecnológicas, pedagógicas y socio-afectivas) a las 
cuales se enfrenta el docente universitario de la UTMACH según su edad. De acuerdo a lo que expresan Melo, López, y Melo (2007) el análisis de varianza a más de gozar de una excelente adaptabilidad a la particularidad de cada diseño, permite que se puedan descomponer las diferentes fuentes de variación, con el fin de analizar una serie de datos que provienen de experimentos aleatorios. Dicho de otra forma, permite analizar si el efecto de interacción entre los dos factores establecidos afecta de manera directa (Pardo, Garrido, Ruiz, y San Martín (2007), o por separado a la variable dependiente (Pardo y San Martín, 2015).

En términos matemáticos simples, el modelo representa la respuesta de $Y_{i j k}$ con los niveles de dos factores A y B, y la interacción entre ambos (AB), resultando según Díaz y Morales (2012); Ruiz (2017) en la siguiente ecuación:

$$
Y_{i j k}=\mu+\alpha_{i}+\beta_{j}+\gamma_{i j}+\epsilon_{i j k}
$$

Donde:

$\mu$, media global sin importar el factor.

$>\alpha_{i}$, es el efecto debido al i-ésimo nivel del factor $A$.

$>\beta_{j}$, es el efecto debido al j-ésimo nivel del factor $B$.

$>\gamma_{i j}$, es el efecto debido a la interacción entre el i-ésimo nivel del factor $A$ y el j-ésimo nivel del factor $B$.

$>\epsilon_{i j k}$, error aleatorio asociado al proceso de muestreo.

\section{Resultados}

\section{Validación del modelo}

En lo que respecta al análisis de ANOVA, para que este tenga validez, es necesario a priori estudiar si las hipótesis básicas del modelo se encuentran o no en contradicción con los datos observados; para cual, se deben satisfacer los supuestos de independencia de ambos factores, normalidad y homocedasticidad (Lara, 2015; Monleón, 2016).

\section{-Independencia}

Para verificar la independencia de los dos factores, se ejecutó una gráfica de dispersión, en donde se tomaron para ser graficados los residuos no tipificados representados en el eje de las ordenadas, y los valores pronosticados no tipificados representado en el eje de las abscisas del modelo ANOVA de dos vías construido. Por lo cual, al observar la Figura 1, se denota en las observaciones que no crean ninguna tendencia curvilínea2; por tanto, no existe interacción entre los dos factores, lo que da paso a que los resultados obtenidos tengan validez interpretativa.

\footnotetext{
${ }^{2}$ Que los datos de los factores formen una línea curva, o mantengan una dirección/relación directamente proporcional.
} 


\section{Figura 1}

Independencia de los factores problemáticas y edad

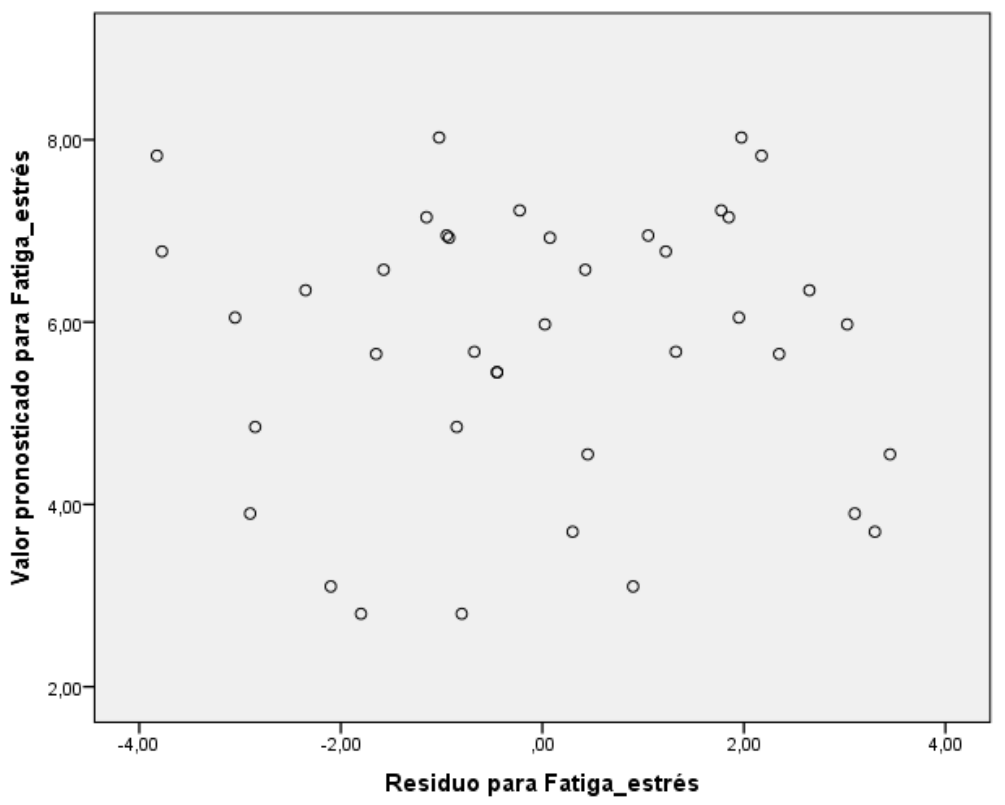

\section{-Normalidad y homogeneidad}

Para efectos de determinar la normalidad se aplicó el test de Shapiro Wilk (González y Cosmes, 2019); y para el cálculo de la homogeneidad de las varianzas tanto del factor edad como del factor problemáticas, se testeó la prueba de Levene (Narváez y Campuzano, 2020). De acuerdo a lo que se visualiza en la Tabla 1, los datos recabados presentan normalidad y homogeneidad de varianzas, debido a que el p-valor de estos se postula por encima del 5\%. Es decir, se aceptan hipótesis nula de existencia de normalidad (Villaseñor, González, y Ochoa, 2017) y de homogeneidad de varianzas para ambos factores (Bustos, Lozano, y Justacaro, 2016).

\section{Tabla 1}

Pruebas de Normalidad y Homogeneidad de Varianzas

\begin{tabular}{cccc}
\hline Normalidad & $\begin{array}{c}\text { Homogeneidad de } \\
\text { varianzas: Edad }\end{array}$ & $\begin{array}{c}\text { Homogeneidad de } \\
\text { varianzas: Problemáticas }\end{array}$ \\
Kolmogorov-Smirnov & Levene & Levene \\
\hline Estadístico & 0.086 & 0.492 & 0.988 \\
p-valor & 0.2 & 0.741 & 0.41 \\
\hline
\end{tabular}

Fuente: Elaboración propia. 


\section{Análisis de varianza doble vía}

Los resultados de la ejecución del modelo ANOVA con dos vías con efectos principales, muestran que los efectos de los factores problemáticas y edad, y los efectos de la intersección, son estadísticamente significativos $^{3}$; es decir, el modelo explica adecuadamente la variación observada en la variable dependiente (nivel de fatiga y estrés); aunque, los dos efectos incluidos en el modelo explican moderadamente la varianza de la variable dependiente, en aproximadamente 36\% (según $\mathrm{R} 2)$.

En consecución, se observa, que el factor problemáticas, posee un $\mathrm{p}>5 \%$, lo que significa, que no existen diferencias estadísticamente significativas entre las cuatro problemáticas que asienten los docentes (logísticas, tecnológicas, pedagógicas y socio-afectivas); lo que se traduce que el nivel de fatiga y estrés por causas de estas problemáticas no se muestran disímiles los unos con los otros. Sin embargo, el factor edad, manifiesta, que los cinco intervalos de edad establecidos demuestran diferencias estadísticamente significativas $(\mathrm{p}<5 \%)$; dicho de otro modo, en que existe uno, o varios intervalos de edad que muestran diferencias respecto a los otros.

\section{Tabla 2}

Salida del modelo ANOVA doble vía

\begin{tabular}{lccccc}
\hline \multicolumn{1}{c}{ Origen } & $\begin{array}{c}\text { Tipo III de } \\
\text { suma de } \\
\text { cuadrados }\end{array}$ & gl & $\begin{array}{c}\text { Media } \\
\text { cuadrática }\end{array}$ & F & Sig. \\
\hline Modelo corregido & $88,725^{\mathrm{a}}$ & 7 & 12,675 & 2,563 &, 032 \\
Intersección & 1334,025 & 1 & 1334,025 & 269,755 &, 000 \\
Problemáticas & 7,875 & 3 & 2,625 &, 531 &, 664 \\
Edad & 80,850 & 4 & 20,213 & 4,087 &, 009 \\
Error & 158,250 & 32 & 4,945 & & \\
Total & 1581,000 & 40 & & & \\
Total corregido & 246,975 & 39 & & & \\
\hline
\end{tabular}

a. $\mathrm{R}^{2}=, 359\left(\mathrm{R}^{2}\right.$ ajustada $\left.=, 219\right)$

Fuente: Elaboración propia.

\footnotetext{
3 Todos aquellos valores de significancia 'p-valor' que sean $<5 \%$, se los considera estadísticamente significativos (Corrales y Dorta, 2019) 


\section{Prueba post hoc}

A vista de los resultados anteriores, al determinar mediante el modelo de ANOVA con dos vías, que los intervalos del factor edad, muestran diferencias estadísticamente significativas, se procedió a aplicar la prueba post hoc de Duncan; test que posee un alto nivel de potencia, y establece un nivel de error por cada prueba, y usa un orden paso a paso para efectuar las comparaciones (Allen, 2017).

Obteniendo así, que el nivel de fatiga y estrés de los docentes universitarios que están dentro del intervalo de edad de 27 a 31 años, con un promedio de 3,38 (más bajo), marcan diferencia estadísticamente significativa respecto a los docentes que poseen edades por encima de 37 años (intervalos de edad 37-41, 42-46, y 47+); no así, con aquellos docentes que oscilan entre 32 y 36 años, ya que la diferencia entre ambos grupos no es significativa, pese a que en términos del promedio del nivel de fatiga y estrés es 1,75 puntos mayor $(5,13)$.

Cabe destacar, que, si bien es cierto, el grupo de profesores de edades comprendidas entre 32 y 36 años, no muestra diferencia estadísticamente significativa en el nivel de fatiga y estrés con los sujetos que están en el intervalo de edad de 27-31 años, este grupo de profesores, trae consigo una condicionante adicional, en el sentido, que pese a ubicarse en el primer subconjunto, también se ubica en el segundo subconjunto, lo que data que tampoco presenta diferencias estadísticamente significativas con aquellos profesores que tienen edades superiores a 37 años.

Adicionalmente, se denota que aquellos educadores que se encuentran entre los 37 y 41 años, son los que mayor nivel de estrés presentan, con un nivel de fatiga y estrés promedio de 7,50.

\section{Tabla 3}

Prueba post hoc de Duncan

\begin{tabular}{lcccc}
\hline & Edad & $\mathbf{N}$ & \multicolumn{2}{c}{ Subconjunto } \\
\cline { 3 - 4 } & & & $\mathbf{1}$ & $\mathbf{2}$ \\
\hline Duncan & 8 & 3,38 & \\
& $32-31$ & 8 & 5,13 & 5,13 \\
& $47+$ & 8 & & 6,25 \\
& $42-46$ & 8 & & 6,63 \\
& $37-41$ & 8 & & 7,50 \\
\hline
\end{tabular}

Fuente: Elaboración propia. 


\section{Gráfico de perfil}

En contraste al inciso predecesor, se aprecia en la Figura $\mathrm{X}$ lo que muestra la prueba post hoc, que el factor de las problemáticas a las que se enfrentan los profesores universitarios en lo referente a los aspectos logísticos, tecnológicos, pedagógicos y socio-afectivos, no muestran diferencia; en cambio, respecto al factor edad, es claramente apreciado que los docentes que se encuentran en edades comprendidas de 27-31 años, manifiestan (según sus respuestas) menor nivel de fatiga y estrés al tener que virtualizar sus clases; caso contrario, con aquellos educadores universitarios, que están en edades de 37 a 41 años, los cuales presentan un nivel de estrés es mayor.

\section{Figura 2}

Gráfico de perfil de las medias marginales de los niveles de fatiga y estrés de los factores problemáticas y edad

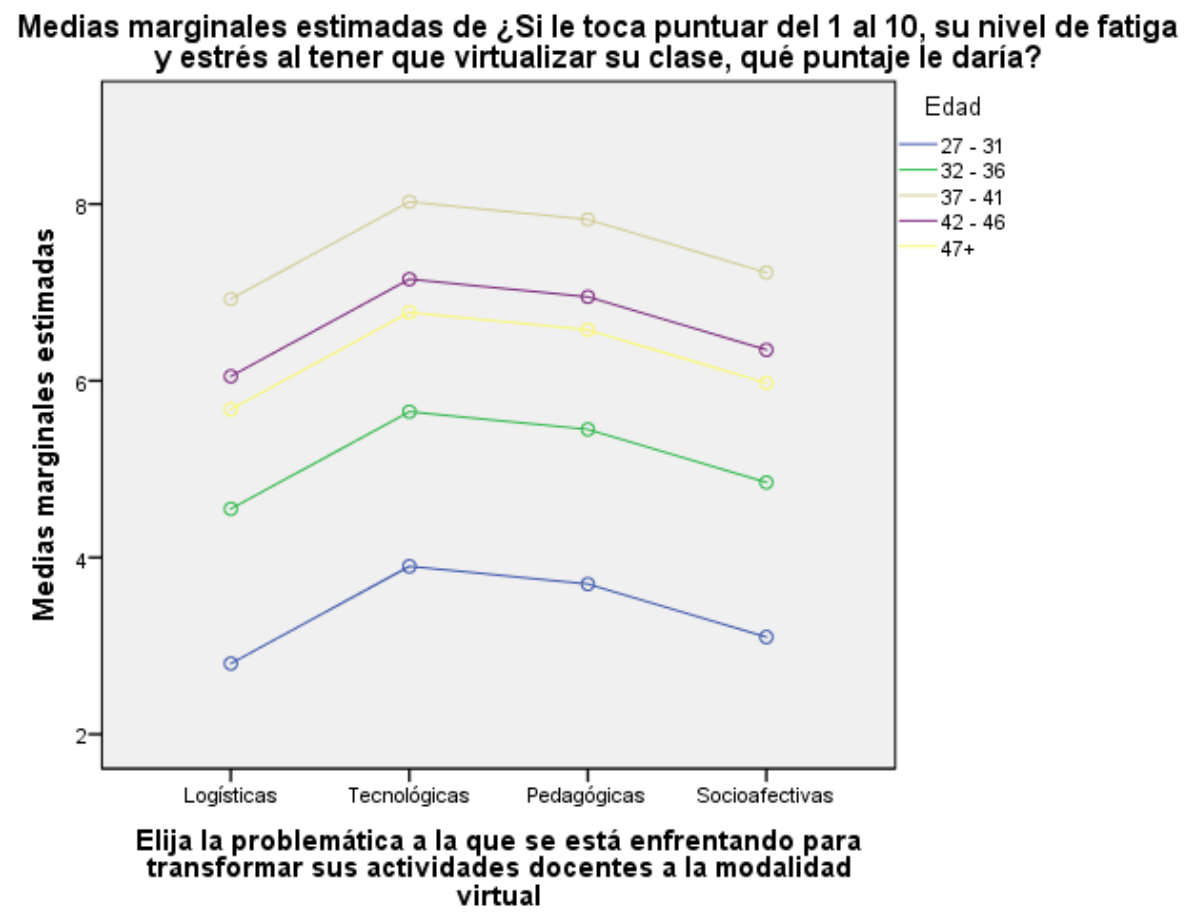

\section{Conclusiones}

En términos generales, los efectos de la pandemia por el COVID-19, implicaron que todos los países del mundo se vean obligados a tomar decisiones radicales y apresuradas, con el claro propósito de contrarrestar el número de casos y muertes registradas desde su aparición en Wuhan; decisiones que no solo afectaron a los factores económicos y de salud pública, sino también, a la 
educación superior (foco de esta investigación), la cual de manera fragosa le tocó migrar de una metodología de enseñanza tradicional presencial, a una enseñanza virtual, causando desazón en la planta docente, al punto de llevarlos a experimentar niveles de fatiga y estrés.

De acuerdo a lo datado por los principales resultados empíricos, exhibidos por el modelo ANOVA, la fatiga y el estrés de los docentes universitarios, no se muestran diferentes en términos de significancia estadística al momento de que los educadores se enfrentan a problemáticas en los aspectos logísticos, tecnológicos, pedagógicos, y socio-afectivos; aunque se denota que, de acuerdo a los valores promedios, se marca (aunque no significativamente) mayor nivel de estrés y fatiga en cuanto a la categoría tecnológica, presumiblemente porque a muchos de estos les tocó aprender a usar las distintas herramientas para adaptar sus contenido a un entorno digital en un corto tiempo.

Finalmente, se halla por el lado de la variable edad, que el nivel de fatiga y estrés en los diferentes rangos establecidos, muestran diferencias estadísticamente significativas, apreciándose principalmente que el menor nivel de estrés radica en los docentes que poseen edades dentro del intervalo de 27 a 31 años; caso disímil sucede con los profesores que tienen edades comprendidas entre 37 y 41 años. Tal situación lleva a plantearse nuevas interrogantes para futuras investigaciones, en donde se consideren variables adicionales que permitan determinar si existe alguna causa que condicione que en el rango de edad señalado es un factor que genere menor nivel o mayor nivel de estrés en los docentes universitarios.

\section{Referencias Bibliográficas}

Alania, R., Llancari, R., de la Cruz, M., y Ortega, D. (2020). Adaptación del cuestionario de estrés académico SISCO SV al contexto de la crisis por COVID-19. Socialium, 4(2), 111-130. https://doi.org/10.26490/uncp.sl.2020.4.2.669

Allen, M. (2017). Post Hoc Tests: Duncan Multiple Range Test. The SAGE Encyclopedia of Communication Research Methods, 1(4). https://dx.doi.org/10.4135/9781483381411.n448

Banco Mundial. (08 de Junio de 2020). La COVID-19 (coronavirus) hunde a la economía mundial en la peor recesión desde la Segunda Guerra Mundial. https://www.bancomundial.org/es/news/press-release/2020/06/08/covid-19-to-plungeglobal-economy-into-worst-recession-since-world-war-ii

Bustos, B., Lozano, R., y Justacaro, G. (2016). Incremento de la fuerza dinámica máxima a través de un protocolo de acción recíproca con deportistas amateurs. Impetus, 10(2), 119-126. http://dx.doi.org/10.22579/20114680.165

Corrales, I., y Dorta, A. (2019). Producción científica en revistas estudiantiles latinoamericanas: análisis comparativo del período 2013-2016. Educación Médica, 20(3), 146-154. https://doi.org/10.1016/j.edumed.2018.02.010

Díaz, L., y Morales, M. (2012). Modelos de doble vía de clasificación. En L. Díaz, y M. Morales, Análisis estadístico de datos multivariados (pág. 155). Bogotá: Universidad Nacional de Colombia. 
Fernández, M., Hernández, D., Nolasco, R., de la Rosa, R., y Herrera, N. (2020). Lecciones del COVID-19 para el sistema educativo mexicano. Iniciativa de educación con equidad y calidad, 1-17.

García, F., Corell, A., Abella, V., y Grande, M. (2020). La evaluación online en la educación superior en tiempos de la COVID-19. Education in the Knowledge Society (12), 1-26. https://doi.org/10.14201/eks.23013

Gómez, N., y Rodríguez, P. (2020). Estrés en docentes en el contexto de la pandemia de COVID19 y la educación. Divulgación académica UNA FENOB, 1(1), 216-234. https://revistas.fenob.una.py/ojs/index.php/rfenob/article/view/91

González, E., \& Cosmes, W. (2019). Shapiro-Wilk test for skew normal distributions based on data transformations. Journal of Statistical Computation and Simulation (17), 1-15. https://doi.org/10.1080/00949655.2019.1658763

Google Noticias. (24 de Agosto de 2020). Google Noticias. https://news.google.com/covid19/map?hl=es-419\&gl=US\&ceid=US\%3Aes-419

Lara, A. (2015). Universidad de Granada (España). Obtenido de https://www.ugr.es/ bioestad/guiaspss/practica7/

Martínez, J., y Garcés, J. (2020). Competencias digitales docentes y el reto de la educación virtual derivado de la COVID-19. Educación y Humanismo, 22(39), 1-16. https://doi.org/10.17081/eduhum.22.39.4114

Melo, O., López, L., y Melo, S. (2007). Diseño de bloques completamente aleatorizados. En O. Melo, L. López, y S. Melo, Diseño de experimentos [Métodos y aplicaciones] (pág. 161). Bogotá: Pro-Offset Editorial S.A.

Monleón, T. (2016). Novedades en el ANOVA. Diseño de experimentos, su análisis y diagnóstico. Technical Report. Universidad de Barcelona. https://www.researchgate.net/profile/Antonio_Monleon-

Getino/publication/304283596_Diseno_de_experimentos_su_analisis_y_diagnostico/link s/576b8cea08aefcf135bd5977.pdf

Mora, A., Julio, M., Calderón, R., y Huilcapi, M. (2020). Enseñar y aprender en tiempos de COVID-19. Pro Sciences: Revista De Producción, Ciencias E Investigación, 4(34), 79-86. https://doi.org/10.29018/issn.2588-1000vol4iss34.2020pp79-86

MSP. (2020). Ministerio de Salud Pública del Ecuador. https://www.salud.gob.ec/actualizacionde-casos-de-coronavirus-en-ecuador/

Narváez, K., y Campuzano, J. (2020). Reformas económicas y su incidencia en el crecimiento del Ecuador, periodo 1980- 2017. MAPA, 4(18), 110-121. http://revistamapa.org/index.php/es/article/view/194/248

Otzen, T., y Manterola, C. (2017). Técnicas de Muestreo sobre una Población a Estudio. International Journal of Morphology, 35(1), 227-232. http://dx.doi.org/10.4067/S071795022017000100037

Pardo, A., y San Martín, R. (2015). Análisis de varianza. Dos factores completamente aleatorizados. En A. Pardo, \& R. San Martín, Análisis de datos en ciencias sociales y de la salud (Vol. II, pág. 247). Madrid: Universidad Autónoma de Madrid. 
Pardo, A., Garrido, J., Ruiz, M. Á., y San Martín, R. (2007). La interacción entre factores en el análisis de varianza: errores de interpretación. Psicothema, 19(2), 343-349. https://www.redalyc.org/pdf/727/72719224.pdf

Pedró, F. (2020). COVID-19 y educación superior en América Latina y El Caribe: efectos, y recomendaciones políticas. Análisis Carolina, 1-15. https://doi.org/10.33960/AC_36.2020

Rodríguez, M., y Mendivelso, F. (2018). Diseño de investigación de corte transversal. Rev.Medica. Sanitas 141-146. https://www.unisanitas.edu.co/Revista/68/07Rev\%20Medica\%20Sanitas\%20213_MRodriguez_et_al.pdf

Rubio, S. (2016). Sección de metodología. Enfermería basada en la evidencia: Investigación clínica aplicada a las ciencias de la salud. Enfermería en Cardiología, 13(69), 23-29. https://dialnet.unirioja.es/descarga/articulo/6285990.pdf

Ruiz, G. (2017). Análisis de varianza. Revista Varianza (13), 54-69. http://www.revistasbolivianas.org.bo/scielo.php?pid=S9876$67892017000100007 \&$ script=sci_arttext

Trujillo, F., Fernández, M., Segura, A., y Jiménez, M. (2020). Escenarios de evaluación en el contexto de la pandemia por la COVID-19. Madrid: Santillana. https://www.researchgate.net/profile/Fernando_Trujillo_Saez/publication/341000931_Es cenarios_de_evaluacion_en_el_contexto_de_la_pandemia_por_la_COVID19_la_opinion_del_profesorado/links/5ea94d0792851cb26763030d/Escenarios-deevaluacion-en-el-contexto-de-1

Villafuerte, J., Johanna, B., Cevallos, Y., y Bermello, J. (2020). Rol de los docentes ante la crisis del COVID-19, una irada desde el enfoque humano. REFCalE, 8(1), 134-150. http://www.refcale.uleam.edu.ec/index.php/refcale/article/view/3214/1986

Villaseñor, J., González, E., y Ochoa, A. (2017). On Testing the Inverse Gaussian Distribution Hypothesis. The Indian Journal of Statistics, 81, 1-15. https://doi.org/10.1007/s13571-017$0148-8$ 